Instituto Internacional de Investigación y Desarrollo Tecnológico Educativo INDTEC, C.A.

DOI: https://doi.org/10.29394/scientific.issn.2542-2987.2017.2.4.8.137-156

OAI-PMH: http://www.indteca.com/ojs/index.php/Revista Scientific/oai

\title{
Estrategias Aplicadas por los Docentes como Gerentes de Aula para el Fortalecimiento de los Aprendizajes
}

\author{
Autor: Franco Javier Jáuregui Contreras \\ Instituto Pedagógico Rural Gervasio Rubio, IPRGR \\ kleijav-@hotmail.com \\ Táchira, Venezuela
}

\section{Resumen}

La presente investigación se ejecutó dentro del paradigma cuantitativo, bajo la modalidad de proyecto factible, con el objetivo de diseñar estrategias dirigidas a docentes para el fortalecimiento de los aprendizajes en los estudiantes de la unidad educativa Arnoldo Gabaldon, ubicada en Delicias, municipio Rafael Urdaneta del estado Táchira. La metodología utilizada responde a las características de la investigación descriptiva de campo, no experimental. La población del estudio estuvo conformada por 72 docentes. Para obtener la información se aplicó, un instrumento contentivo de 20 ítems, los cuales midieron los factores que inciden la aplicación de estrategias gerenciales para el fortalecimiento de los aprendizajes en los estudiantes. Este instrumento fue validado y se obtuvo un índice de confiabilidad de 0,81. En el análisis de resultados se determinaron las frecuencias simples y absolutas de cada reactivo. Lo cual, dio como resultado una serie de situaciones acontecidas constantemente que permitió llegar a las siguientes conclusiones: las estrategias gerenciales son empleadas medianamente por los docentes, como también en la organización y la planificación, no existe un compromiso en relación a ello, aunque allí se logró detectar que los docentes cumplen medianamente con las actividades planificadas. Además de ello en el caso de la dirección y control se denota como de manera contundente se cumple con ambos procesos, incluso de manera mayoritaria, y ambos se asumen como estrategias gerenciales. Por lo tanto, se recomienda la aplicación de las estrategias.

Palabras clave: estrategias; docentes; gerentes de aula; aprendizajes. 


\title{
Strategies Applied by Teachers as Classroom Managers to Strengthen Learning
}

\begin{abstract}
The present research was executed within the quantitative paradigm, under the feasible project modality, with the objective of designing strategies aimed at teachers to strengthen learning in the students of the Arnoldo Gabaldon educational unit, located in Delicias, Rafael Urdaneta municipality State Táchira. The methodology used responds to the characteristics of field descriptive research, not experimental. The study population consisted of 72 teachers. To obtain the information, a 20 -item contentive instrument was applied, which measured the factors that influence the application of managerial strategies to strengthen student learning. This instrument was validated and a reliability index of 0.81 was obtained. In the analysis of results, the single and absolute frequencies of each reagent were determined. This resulted in a series of constantly occurring situations that led to the following conclusions: managerial strategies are employed by teachers on average, as well as in organization and planning, there is no commitment in relation to it, although there It was possible to detect that teachers comply with the planned activities on a regular basis. In addition, in the case of management and control, it is denoted how forcefully both processes are fulfilled, even in the majority, and both are assumed as managerial strategies. Therefore, the implementation of strategies is recommended.
\end{abstract}

Keywords: strategies; teachers; classroom managers; learning. 


\section{Introducción}

El hombre es un ser social que depende en gran parte de sus semejantes para lograr el desarrollo integral de sus potencialidades, su relación con los demás está caracterizada por la formación obtenida en su familia y en la educación de la sociedad, por ello, una de las metas de la educación a nivel mundial está relacionada con la formación integral del hombre, para así ofrecer ideas y posibles soluciones a situaciones suscitadas en momentos inesperados, tales como, aquellos provocados por fenómenos naturales o por razones de índole personal (salud, social, económicas, entre otros) que desvirtúan el normal desenvolvimiento de las actividades planificadas, así desde esta perspectiva, dicen Leithwood y Louis (1998: 3), "que la imagen de las escuelas como organizaciones parece ser un respuesta prometedora a las continuas demandas de reconversión de la educación".

En los actuales momentos, Venezuela construye un modelo de desarrollo para el cual, Así, la calidad de la educación de un país está determinada por la calidad de sus docentes, que estén orientados hacia la búsqueda de la excelencia, de manera que puedan enseñar al estudiante a ser, a aprender, a convivir y a hacer. Es por ello que la práctica docente incorpora al quehacer diario estrategias que permiten a los estudiantes desarrollar el conocimiento de forma sencilla y eficaz. Las cuales se diseñan y ejecutan con la finalidad de fortalecer los contenidos que casi siempre a una finalidad, aunque quizá no siempre se desarrollan a nivel consciente y deliberado.

En tal sentido, el docente como gerente de aula debe diseñar las estrategias necesarias y adecuadas para desarrollar las actividades académicas tomando en cuenta el área a tratar y las condiciones en que se encuentran los estudiantes, las estrategias permiten identificar principios, criterios y procedimientos que configuran la forma de actuar del docente en relación con la programación, implementación y evaluación del proceso de 
enseñanza aprendizaje. Evitando que el proceso se convierta en un simple suministro de información mecánico y por demás vacío que no deja de incrementar el desinterés en las aulas. De allí, que habría que empezar comprendiendo en qué consiste el proceso de enseñanza y aprendizaje, y para ello Méndez, (2004) señala, "es el conjunto de acciones dirigidas al logro de un aprendizaje significativo y constructivo, que involucra qué es lo que se aprende y cómo se aprende” ... (pág. 42). Es conveniente, para llevar a cabo este proceso se requiere de cierta preparación, que si bien es cierto que los docentes están preparados para ello, es inevitable sorprendernos cada día por el deterioro de la calidad de estudiantes que cada año muestran las estadísticas.

Así bien la aplicación de todas las funciones permitirá alcanzar buenos resultados en la práctica docente dentro y fuera del aula de clase, ya sea con la comunidad y las organizaciones que hacen vida allí, estas siempre deben estar ligadas a los recursos de enseñanza aprendizaje de manera tal que se logre el aprendizaje significativo.

A partir de esto, el docente como gerente de aula, tiene que poner en práctica una planificación educativa, donde genere la mayor cantidad de oportunidades de participación e interacción para los estudiantes, lograr el éxito en el alcance de las metas propuestas, así como también reducir el impacto del cambio, minimizando el desperdicio y estableciendo criterios utilizados para controlar.

\section{EI Problema}

La calidad de la educación depende principalmente del docente, de la forma en que cumpla con las funciones administrativas conocidas como: planificación, organización, dirección y control, que conduzcan al crecimiento personal, ético y creativo del estudiante; de la forma en que se comprometa a estudiar profundamente la realidad social del país y a desempeñar el 
verdadero papel de Educar. Dentro del esquema educativo, Ruiz (2002) plantea que el docente juega un papel importante, al ser el agente transformador de esta sociedad, por una sociedad más justa, más humana, más creativa; de allí que se requiere que sea: un guía, orientador, facilitador, investigador, motivador, participativo y creador de oportunidades que contribuyan al proceso de enseñanza y aprendizaje, fomentando la utilización de técnicas y estrategias de enseñanza que estimulan las actividades académicas en base a las necesidades e inquietudes del estudiante.

Según Méndez (2004) es evidente que muchas cosas de las que hace un docente en el aula deben depender del tipo de ambiente en el que trabaja. Es decir, que por ser el aula ese especial ambiente de trabajo, el docente o gerente de esa aula está condicionado por algunas características típicas del aula y de la institución en la que se desenvuelve. Lamentablemente algunos docentes se acostumbran a mirar al aula como un cuarto estanco de cuatro paredes, que reúne estudiantes para aprender un determinado tema, la verdad es que ciertos docentes no conocen su ambiente de trabajo a profundidad, su real dimensión.

A tal efecto, Salazar (2004) expresa que la gerencia en el aula se está desarrollando de tal forma que algunos docentes no asumen el rol de facilitador pues no están creando un ambiente o clima inicial para la experiencia a desarrollar en clase, poco estimulan o ayudan a despertar y esclarecer los propósitos de los alumnos, así como los objetivos más generales del grupo, no se confía en que el estudiante desea realmente alcanzar aquellas metas significativas para él, siendo éste la fuerza motivacional que subyace en todo aprendizaje, no se organiza y se pone a disposición de los alumnos, la más amplia y variada gama de recursos para el aprendizaje y poco se considera a sí mismo como un recurso flexible que estará en disposición de prestar todo tipo de ayuda al grupo. Dentro de este orden, el docente como gerente, necesita combinar las estrategias, técnicas y 
recursos disponibles de manera eficiente, para facilitar el logro de los objetivos trazados en el proceso, en forma conjunta docente y estudiante.

Esta situación se ha venido observando en los últimos meses en la Unidad Educativa Arnoldo Gabadón, ubicado en delicias, Municipio Rafael Urdaneta. En tal sentido, es importante que el docente haga una revisión de las practicas pedagógicas que emplea en el aula de clase y reflexione sobre la manera cómo hasta ahora ha impartido los contenidos, para que de esta manera pueda conducir su enseñanza con técnicas y recursos adecuados que le permitan al educando construir de manera significativa el conocimiento y alcanzar el aprendizaje de una forma efectiva. Es por ello la importancia que los educadores asuman con responsabilidad el proceso de enseñanza, facilitándole al educando las herramientas que le permitan nuevas y reales metas, siendo éste apoyo fundamental para marcar la diferencia.

Por esta razón se proponen estrategias dirigidas al docente para fortalecer y mejorar las formas de aprendizaje en los estudiantes en situaciones de interrupciones académicas. Por tal motivo, se pretende mediante esta investigación dar respuesta a las siguientes interrogantes: ¿Qué conocimiento tienen los docentes acerca de las diferentes estrategias de aprendizaje que aplica? ¿Las estrategias utilizadas por los docentes son aplicadas correctamente? ¿Qué conocimiento tiene de los nuevos lineamientos curriculares? Y ¿Cuál es la importancia del uso de las estrategias de aprendizaje? Esta, entre otras interrogantes que se podrán generar de la dinámica del estudio.

\subsection{Objetivos}

\subsubsection{Objetivo General}

Determinar las estrategias aplicadas por los docentes como gerentes de aula para el fortalecimiento de los aprendizajes, en los estudiantes de la 
Unidad Educativa Arnoldo Gabaldón, (Delicias, municipio Rafael Urdaneta del Estado Táchira).

Por otra parte se tomaron como antecedentes del estudio los siguientes trabajos de investigación: A nivel nacional, es conveniente señalar la investigación realizada por Urdaneta (2005), que consiste en una tesis Titulada "Actuación del Docente como gerente en la I Etapa de Educación Básica" cuyo objetivo de estudio se refiere a caracterizar la actuación del docente como gerente en la, Primera Etapa de Educación Básica, enfocada básicamente a determinar el grado de manejo y conocimiento de las prácticas gerenciales en todas las actividades desarrolladas en la institución. Reflejó igualmente, el conocimiento que los docentes investigados poseen en cuanto; a los aspectos normativos del proceso gerencial que demanda la Reforma Educativa del Currículo Básico Nacional.

Martínez (2007) planteó un "Modelo de Gerencia Participativa Mediante Compromiso y Conservación de la Planta Física del NER 126 del Municipio Rafael Urdaneta del estado Táchira", la metodología aplicada se basó en una investigación cualitativa etnográfica, con aplicación de encuestas, entrevistas y observaciones directas aplicadas a la población objeto de estudio conformada por directora, docentes, alumnos, representantes y miembros de la comunidad. La información fue recabada por tres técnicas: (1) observación participativa, donde la observadora compartió vivencias con las personas en estudio, tomando notas de campo; (2) entrevistas en profundidad aplicada a los diferentes sectores relacionados con la escuela, por medio del diálogo sencillo, informal y sin orden de preguntas prefijadas; y (3) cuestionarios abiertos, se analizaron por medio de porcentajes y gráfico. Los resultados obtenidos permitieron concluir que el mantenimiento de la planta física generalmente lo realizan los docentes y alumnos, en ocasiones los representantes colaboran para comprar materiales, otros limpian la maleza o pintan la escuela, de igual manera, la carencia de diálogo entre la directora, 
docentes y comunidades sobre el problema de la planta física de la institución no contribuye en la integración escuela-comunidad.

Este antecedente se relaciona con el estudio, en virtud de que plantea la participación activa de la comunidad y el compromiso que los actores educativos deben tener para que el quehacer comunitario se vea fortalecido.

\section{Bases Teóricas}

Toda organización requiere de la gerencia, que radica básicamente en planificar, organizar, controlar y dirigir los recursos, para el logro de los objetivos. De allí que el gerente es la persona o individuo que logra metas $u$ objetivos por medio de otras personas, a través de un proceso de toma de decisiones, distribución de recursos y dirección de actividades. Al respecto, Ruiz (2002) expresa:

El término gerencia se refiere a las organizaciones que efectúan actividades de planificación, organización, dirección y control a objeto de utilizar sus recursos humanos, físicos y financieros con la finalidad de alcanzar objetivos comúnmente relacionados con beneficios económicos. (pág. 27).

Por otra parte, Marsh (2003) considera "la gerencia como un arte, una ciencia que se ocupa del uso correcto, provechoso y sistemático de todos los recursos de una empresa (pág. 35)". Por estas razones, el término gerencia ha sido de especial interés, por todo lo que implica, cabe señalar que en un principio, dicho término estaba relacionado exclusivamente con las organizaciones con fines de lucro, sin embargo con el paso de los años este término fue adoptado por otros tipos de organizaciones, entre ellas las educativas.

Bob y Peter (2002) manifiestan que "la gerencia consiste en lograr que se hagan las cosas por medio de los demás... hacer que algo planeado suceda en un área específica por medio de los recursos disponibles" (pág. 9-10). 
Se puede aclarar que la gerencia es ejercida por una persona que tiene a su cargo el buen funcionamiento de una institución y que cumple las siguientes fases: planificación, organización, dirección, ejecución y evaluación, con el fin de alcanzar objetivos y metas propuestas, La planificación es la más importante de las funciones de un gerente de aula, planificar es determinar anticipadamente que es lo que se va a hacer, todo proceso administrativo se inicia con una planificación, ya que es aquí donde se determinan los objetivos y la mejor forma de ser alcanzados; para Koontz y O'Donnell (2002), "la planificación consiste en la selección de misiones y objetivos y acciones para lograrlos; requiere toma de decisiones, es decir, seleccionar recursos futuros de acción entre varias opciones." (pág. 45).

Por otra parte, la planificación es la función a través de la cual el docente organiza las actividades, conocimientos, habilidades, destrezas que deberán adquirir o realizar los educandos. En otro orden de ideas, la organización es la segunda etapa del proceso gerencial, que consiste básicamente en determinar las actividades que se realizarán, quienes las realizarán y de qué forma. Al respecto, Robbins (2003) expresa como "la organización incluye la determinación de las tareas que se realizarán, quién las hará, cómo se agruparán las labores y quién reportará a quién y dónde se tomarán las decisiones." (pág. 114). A tal efecto, organizar es el proceso de determinar y establecer la estructura, los procedimientos y los recursos necesarios para lograr de los objetivos establecidos en la planificación. De allí que el docente debe seleccionar junto con los educandos las actividades, definir las actividades y sus responsables, entre otras.

Asimismo, la dirección, es el elemento de la administración en el que se logra la realización efectiva de todo lo planeado, por medio de la autoridad del administrador, ejercida a base de decisiones, ya sea tomada directamente, y con más frecuencia. Por su parte, Koontz y O’Donnell (2002) afilian el término dirección como "La función ejecutiva de guiar y vigilar al subordinado." (pág. 
114). Por consiguiente, la parte esencial y central de la organización, a la cual se debe regir todos los elementos es precisamente la dirección. En este orden el educador debe dirigir de un modo muy apropiado.

Finalmente, el control en el transcurso de enseñanza básicamente está determinado por el proceso de evaluación, ésta es la función que consiste en verificar el logro de los objetivos planteados en la planificación, al respecto Ruiz, (2002) señala que "La evaluación permite controlar las capacidades de los alumnos en cada uno de los momentos del desarrollo de la estrategia." (pág. 85).

Por otra parte, la gerencia de aula también puede ser definida como un proceso de planificación, organización, dirección y control de las actividades de aprendizaje implícitos en un diseño curricular. En consecuencia, el docente como gerente de aula va ejercer las funciones administrativas relacionándolo con los recursos de enseñanza-aprendizaje, de manera tal que se logre el aprendizaje significativo.

De igual forma, el docente debe poner en práctica su creatividad para diversificar la enseñanza, pues con un poco de imaginación los trabajos rutinarios los puede transformar en actividades desafiantes para el estudiante.

En consecuencia, El Ministerio del Poder Popular para la Educación (2007) plantea que la planificación en el sistema educativo bolivariano es dinámica y flexible, en ella se organizan los elementos que conforman el proceso de aprendizaje, tomando en consideración los resultados del diagnóstico pedagógico integral, el perfil del ciudadano y ciudadana a formar, finalidades de las áreas de aprendizaje, componentes, intencionalidades, ejes integradores, estrategias y recursos de aprendizajes, indicadores, estrategias, instrumentos, tipos y formas de evaluación.

Se desarrolla a través de las formas de organización: Proyecto Educativo Integral Comunitario (PEIC), la Clase Participativa (CP), Proyectos 
de Aprendizaje (PA), Proyectos de Desarrollo Endógeno (PDE) y el Plan Integral (PI).

\subsection{Bases Legales}

En los fundamentos legales están contenidos las normativas por la cual se rige el país y su sistema educativo. A tal efecto se presenta a continuación algunas de los referentes legales que sustentan el estudio.

Constitución de la República Bolivariana de Venezuela (1999).

Artículo 102. 103. Y 104 (CRBV) y LOE (2009).

\section{Metodología}

Esta investigación se ubicó en la modalidad de proyecto factible, el cual según la Universidad Pedagógica Experimental Libertador (2006). La define como "la elaboración y desarrollo de una propuesta de un modelo operativo viable para solucionar problemas, requerimientos 0 necesidades de organizaciones o grupos sociales" (pág. 35). Al respecto, en el presente estudio propuso estrategias dirigidas al docente para fortalecer su actitud hacia el desarrollo de las formas de aprendizaje en la Unidad Educativa Arnoldo Gabaldón, ubicado en delicias, municipio Rafael Urdaneta, Estado Táchira.

Así mismo, el estudio se apoyó en una investigación de campo de naturaleza descriptiva; para lo cual Palella (2006) señala que es "el de interpretar realidades de hecho. Incluye descripción, registro, análisis e interpretación de la naturaleza actual, composición o procesos de los fenómenos". Por ello, se determinará las estrategias aplicadas por los docentes como gerentes de aula para el fortalecimiento del conocimiento no adquirido, el impacto de cada una de las estrategias aplicadas por los docentes como gerentes de aula y las formas de organización de los aprendizajes según los nuevos lineamientos curriculares en las que el docente como gerente de 
aula, debe aplicar nuevas estrategias metodológicas para instruir sobre los contenidos o conocimientos no adquiridos.

En cuanto a la investigación de campo, Balestrini (2002) señala que "la investigación de campo permite establecer la interacción entre los objetos y la realidad de la situación de campo, observar y recolectar los datos directamente de la realidad en la situación natural" (pág. 37); es decir, los datos serán tomados de una realidad concreta donde el autor del estudio interactuara directamente con los docentes de la Unidad Educativa Arnoldo Gabaldón, ubicado en delicias, municipio Rafael Urdaneta, Estado Táchira.

\subsection{Diseño de la Investigación}

\subsubsection{Fases del Estudio}

Por ser un proyecto factible la investigación se desarrollará cumpliendo las siguientes fases: diagnóstico, factibilidad y diseño.

\subsubsection{Fase Diagnóstica}

En esta fase se determinará la situación actual con referencia al uso de estrategias aplicadas por los docentes como gerentes de aula para el fortalecimiento de los aprendizajes en los estudiantes de la Unidad Educativa Arnoldo Gabaldón, ubicado en delicias, municipio Rafael Urdaneta, Estado Táchira.

4.1.1.2. Población y Muestra. El presente estudio la población estuvo tomada en su totalidad (35) docentes

4.1.1.3. Instrumento de Recolección de Datos. En la presente investigación el autor del estudio diseñara un instrumento tipo cuestionarios el cuales tienen como finalidad recabar información referida a estrategias aplicadas por los docentes para fortalecer los aprendizajes en los estudiantes de la Unidad 
Educativa Arnoldo Gabaldón, ubicado en delicias, municipio Rafael Urdaneta, Estado Táchira.

\subsubsection{Validez y Confiabilidad del Instrumento.}

La validez de contenido que se realizó a los instrumentos se llevó a efecto a través de la técnica "juicio de expertos", para lo cual fueron seleccionados tres Magíster en Educación de los cuales uno es especialista en el área metodológica

Seguidamente, se utilizó el estadístico Alpha de Cronbach a través su fórmula:

Una vez aplicada la fórmula, se obtuvo un coeficiente de confiabilidad de 0,81 para los docentes considerada según el criterio de Ruiz (2002) como una magnitud muy alta.

4.1.1.5. Procedimiento y Análisis de los Datos. Luego de la aplicación del instrumento a la muestra para recolectar la información se procedió a la elaboración en forma manual de una matriz de datos, en donde se transcribieran las respuestas dadas por los sujetos de estudio a cada uno de los ítems, lo cual permitirá determinar la frecuencia relativas y absolutas para cada alternativa de respuesta, con el apoyo del programa estadístico SPSS.

\subsubsection{Fase de Factibilidad}

En esta fase se determinaron los siguientes aspectos: legal, institucional, técnico y económica.

\subsubsection{Fase de Diseño}

Una vez obtenidos los resultados del diagnóstico se procederá a diseñar Estrategias dirigidas al docente como gerentes de aula para el fortalecimiento del conocimiento no adquirido en los estudiantes de la Unidad Educativa 
Arnoldo Gabaldón, ubicado en Delicias, municipio Rafael Urdaneta, Estado Táchira.

\subsection{Análisis y Presentación de los Resultados}

\subsubsection{Estrategias Gerenciales Aplicadas por los Docentes como} Gerentes de Aula.

En definitiva, las estrategias gerenciales son empleadas medianamente por los docentes objeto de estudio, en el caso de la organización y la planificación el manejo es mediano, no existe un compromiso en relación a ello, igual situación se refleja en la organización, aunque allí se logró detectar que los docentes cumplen medianamente con las actividades planificadas. Además de ello en el caso de la dirección y control se denota como de manera contundente se cumple con ambos procesos, incluso de manera mayoritaria, y ambos se asumen como estrategias gerenciales.

\subsubsection{Formas de Aprendizaje}

El procedimiento a seguir en la ejecución de las estrategias es la del taller de carácter vivencial, en la que los participantes reflexionan y generan a su vez los contenidos tratados con nuevos patrones sustentados en la orientación al cambio en el desempeño de la acción en el aula. Así mismo, la realimentación, la comunicación y la participación espontánea constituyen un aporte fundamental en la dinámica de cada situación en pro de resultados significativos para quienes internalicen en cada una de las jornadas planificadas que a su vez implican actividades de introducción por parte del docente. 


\section{Conclusiones y Recomendaciones}

\subsection{Conclusiones}

Con respecto al objetivo específico $\mathrm{N}^{\circ} 1$, que hace referencia a: Diagnosticar las estrategias de enseñanza aplicadas por los docentes como gerentes de aula. En definitiva, las estrategias gerenciales son empleadas medianamente por los docentes objeto de estudio, en el caso de la organización y la planificación el manejo es mediano, no existe un compromiso en relación a ello, igual situación se refleja en la organización, aunque allí se logró detectar que los docentes cumplen medianamente con las actividades planificadas

En cuanto al objetivo específico $\mathrm{N}^{\circ} 2$, que hace referencia a: Precisar las funciones gerenciales que aplica el docente como gerente de aula. se puede señalar lo siguiente de acuerdo a los resultados obtenidos: se evidencia la falta de integración del personal docente, es decir, de una mayor participación e integración de los mismos en la planificación de las formas de aprendizaje, específicamente en el PEIC, pocas veces definen estrategias de gestión escolar, integrando los principios: pedagógicos, políticos, socioculturales y comunitarios; es definida la visión y misión de la escuela en el PEIC durante el proceso de contextualización de finalidades. Asimismo, el PEIC de la institución en algunas ocasiones implica la observación e investigación, planificación, coordinación, para la ejecución y evaluación de todas aquellas acciones previstas para lograr los objetivos propuestos a nivel: académico, administrativo y comunitario y gestiona acciones que permiten atender necesidades detectadas, fortalecer las experiencias alcanzadas e incorporar nuevas propuestas que propicien las transformaciones requeridas.

Finalmente, con respecto a los planes integrales se evidencia que pocas veces el personal docente a través de estos logra profundizar aspectos abordados sutilmente en los proyectos que requieran niveles más altos de apropiación y abstracción, asimismo, en algunas ocasiones aborda este tipo 
de planes con la finalidad desarrollar potencialidades que son esenciales para el logro de otras de mayor complejidad.

Es necesario resaltar; que el docente de aula es el responsable de mediar el cumplimiento de los objetivos propuestos, así como velar por la comprensión de significados a través de la implementación de actividades estratégicas (intercambio de ideas, experiencias significativas) que coadyuven al estudiante, en la búsqueda de conocimientos a través de ambientes agradables.

Cabe resaltar; que la disposición al cambio por parte del docente hacia las nuevas formas de aprendizaje, es donde se encuentra la base para el logro de un producto escolar de calidad, por cuanto va a ser el moderador de expectativas conducentes al incentivo y puesta en práctica de actividades generadoras de su propio conocimiento significativo.

\subsection{Recomendaciones}

Realizar diagnósticos de recursos disponibles en la institución y en la comunidad antes de abordar formas de organización de los aprendizajes, así como de los intereses y necesidades de los miembros de la comunidad educativa.

Mantener comunicación permanente entre los miembros de la Unidad Educativa, representantes y estudiantado.

Ejecutar estrategias donde participen activamente docentes, estudiantes y comunidad educativa en forma integrada, con compromiso desde el inicio hasta el final de la actividad planificada.

Entender que las formas de organización de los aprendizajes en los centros escolares, encuentra una forma de ser, de hacer y aportar, un medio para la consecución de una mejor calidad de vida, una opción para una mejor educación, una estrategia para el logro de aprendizajes significativos. 


\section{Propuesta}

\subsection{Estrategias de Enseñanza Dirigidas al Docente para Fortalecer los} Aprendizajes en la Unidad Educativa Nacional Arnoldo Gabaldon. Ubicada en Delicias, Municipio Rafael Urdaneta, Estado Táchira.

Con el transcurrir del tiempo, el hombre se ha visto en la necesidad de organizarse en el desarrollo continuo de sus actividades, con la finalidad de cumplir sus metas y objetivos. Es por ello que se ha visto en la necesidad de aplicar estrategias, las cuales se conciben como un conjunto de destrezas y habilidades orientadas (de lenguaje, concordancia, discordancia y grupales) que deben ser internalizadas y asimiladas por los docentes para fortalecer su rol como gerentes educativos.

Esto ofrecerá al estudiantado ventajas indiscutibles que le permitirán tener un mayor aprovechamiento de la enseñanza, pues la misma le va a brindar la posibilidad de estar en contacto con el aprendizaje en cualquier momento que así él disponga.

\subsection{Objetivos de la Propuesta}

\subsubsection{Objetivo General}

Proponer a los docentes como gerentes educativos un conjunto de estrategias que le permitan desarrollar destrezas y habilidades, para el fortalecimiento de los aprendizajes en la gestión escolar.

\subsubsection{Objetivos Específicos}

1. Propiciar integración armónica entre el facilitador y los participantes, a través de mesas de trabajo y a su vez se discutan conocimientos básicos sobre gestión escolar, habilidades de un gerente, cualidades y funciones.

2. Destacar la importancia de las formas de organización de los aprendizajes e Instruir a los docentes acerca de la importancia que tiene 
el cómo se organiza el PEIC, la clase participativa y el diseño de la planificación a corto, mediano y largo plazo.

3. Sensibilizar a los participantes con la necesidad de abordar responsablemente el proyecto endógeno como una forma de organización de los aprendizajes.

4. Concienciar a los participantes sobre la importancia de de desarrollar acciones que incorporen los planes integrales para fortalecer el proceso de planificación escolar.

\section{Referencias}

Balestrini, M. (2002). Cómo se elabora el proyecto de investigación. Caracas: Consultores asociados.

Bob, L. y Peter, B. (2002). Gerencia. Bogotá: Norma.

Constitución de la República Bolivariana de Venezuela (1999). Gaceta oficial № 36860. Diciembre 30 de 1999. Venezuela.

Méndez, M. (2004). Gerencia de aula en el proceso de enseñanzaaprendizaje en la asignatura sistemas operativos en el área de aduanas. trabajo de grado en maestría en educación. Universidad de Carabobo (área de estudios de postgrado).

Marsh, L. (2003). Las Organizaciones. México. McGraw-Hill.

Martínez, S. (2005). Modelo de Gerencia Participativa Mediante Compromiso de las Comunidades Educativas en la Gestión Escolar. Trabajo de Grado no Publicado. UPEL Rubio.

Ministerio del Poder Popular para la Educación (2007). Material Mimeografiado. La Planificación Educativa. Sistema Educativo Bolivariano. Caracas.

Palella, S. y Martins, S. (2006). Metodología de la investigación cualitativa. Caracas; FEDUPEL.

Ruiz, L. (2002). Gerencia en el aula. Venezuela: FEDUPEL. 
Robbins, S. (2003). Administración, teoría y Práctica. México: Prentice Hall Hispanoamerica, S.A.

Salazar, J. (2004). Gerencia de Aula. Trabajo de grado entregado para publicación. (UPEL). Venezuela: Lara.

Universidad Pedagógica Experimental Libertador, Vicerrectorado de Investigación y Postgrado. (2006). Manual de trabajo de grado de especialización, maestría y tesis doctorales. Caracas: Autor.

Urdaneta, F. (2005). Actuación del Docente como Gerente en la I Etapa de Educación Básica. Trabajo de Grado No Publicado. UPEL. 


\section{Franco Javier Jáuregui Contreras \\ e-mail: kleijav-@hotmail.com}

Venezolano, domiciliado en delicias municipio Rafael Urdaneta del Estado Táchira, Venezuela. Graduado en Educación Integral UPEL 2002, con especialización en Evaluación educacional en Valle de Monboy 2005, maestría en Gerencia Educacional UPEL-2012, Actualmente realiza Estudios de

Doctorado en Educación en la UPEL-Rubio. Actualmente se encuentra inscrito en el núcleo de investigación: FIPSED. En la línea de Investigación educación. Se ha desempeñado en el MPPE como docente de Aula. Coordinador de proyectos productivos, coordinador institucional, Coordinador Municipal del PAE. Director del NER 294 y actualmente se desempeña como Supervisor Educativo en el Circuito Escolar № 2 del Municipio Rafael Urdaneta.

El contenido de este manuscrito se difunde bajo una Licencia de Creative Commons Reconocimiento-
NoComercial-Compartirlgual 4.0 Internacional 